\title{
AVALIAÇÃO DA CAPACIDADE RESPIRATÓRIA DE SERVIDORES DE BIBLIOTECAS DE UMA UNIVERSIDADE PÚBLICA
}

\author{
EVALUATION OF THE RESPIRATORY CAPACITY OF \\ LIBRARY SERVERS OF A PUBLIC UNIVERSITY
}

Jessica Hosana de Paula (Orcid: 0000-0003-3855-0879) ${ }^{1}$ Luiza Grassmann Dias (Orcid: 0000-0003-2460-9438) Ricardo Rasmussen Petterle (Orcid: 0000-0001-7735-1077) Ângela Luiza Cunha Legey (Orcid: 0000-0002-4295-4182) João Adriano de Barros (Orcid: 0000-0001-5043-6652) Arlete Ana Motter (Orcid: 0000-0002-2585-207X)

Contato

Jessica Hosana de Paula

E-mail: jessicahosanar@gmail.com

${ }^{1}$ Curso de Fisioterapia, Universidade Federal do Paraná, Paraná, Brasil. ${ }^{2}$ Curso de Estatística; Programa de Pós-Graduação em Saúde Coletiva, Universidade Federal do Paraná, Paraná, Brasil.

${ }^{3}$ Curso de Medicina, Universidade Federal do Paraná, Paraná, Brasil

\section{RESUMO}

Objetivo: avaliar a capacidade respiratória de servidores de bibliotecas de uma universidade pública. Método: A amostra foi composta por 28 participantes. A avaliação respiratória foi composta por manovacuometria, espirometria pré e pós-broncodilatador, teste da caminhada de 6 minutos (TC6) e escala de dispneia Borg modificada. Na análise estatística, foi utilizado teste Shapiro-Wilk, Teste de Wilcoxon, Teste Exato de Fisher e a correlação de Spearman realizadas no software R e no SPSS ${ }^{\oplus}$.0. Resultados: Em sua maioria, a amostra foi composta por mulheres, com média de idade 37,9 anos, obesas, nível médio de escolaridade, não fumantes e praticantes de atividade física. Na manovacuometria, 5 participantes (17,86\%) obtiveram PImáx normal; e na PEmáx, 9 participantes $(32,14 \%)$ foram classificados com valores de normalidade. Ao correlacionar a PImáx e a PEmáx com a espirometria, houve relação com PFE litros pré e pós-broncodilatador (correlação de Spearman p $\geq 0,05$ ). Conclusões: A maioria dos participantes não atingiu a distância prevista no teste de caminhada de 6 minutos. Houve diferença significativa entre a escala de dispneia de Borg aplicada antes e depois do TC6. Na espirometria, 89,3\% da amostra obteve espirometria normal; e 10,7\%, obstrução média.

Palavras-chave: Saúde do trabalhador; Fisioterapia; Testes de Função Respiratória.

\section{ABSTRACT}

Objective: The aim of the study was to evaluate the respiratory capacity of library servers of a public university. Method: (colocar tipo do estudo) The sample consisted of 28 participants. Respiratory evaluation was performed through manovacuometry, before and after the use of a bronchodilator, spirometry, 6 minute walk test, and modified Borg dyspnea scale. Statistical analysis used the Shapiro-Wilk test, Test Wilcoxon, Test Exato de Fisher e a Spearman correlation test in the SPSS 2.0 software R. Results: Most of the sample consisted of women, with an average age of 37.9 years, obese, middle-level, non-smokers and physical activity practitioners. Conclusion: Most participants did not complete the 6-minute walk test. There was a significant difference between the Borg dyspnea scale applied before and after the $6 \mathrm{MWT}$ (colocar numeros), $89.3 \%$ of the participants had a normal spirometry and $10.7 \%$ mean obstruction.

Keywords: Health evaluation; physical therapy specialty; Respiratory Function Tests. 


\section{INTRODUÇÃO}

As Doenças Crônicas Não Transmissíveis (DCNT) protagonizam o maior problema global de saúde no mundo. Segundo dados da Organização Mundial da Saúde (OMS), cerca de 3,6 milhões de pessoas morreram devido às DCNT. Entre as principais causas, estão as doenças do aparelho circulatório, o diabetes, o câncer e as doenças respiratórias ${ }^{1}$, foco deste estudo.

As doenças respiratórias são provocadas por reações alérgicas, infecções, inalação de poeiras ou produtos químicos, exposição em ambiente insalubres, por quedas súbitas na temperatura ou por aumento em níveis de poluentes. Os principais sintomas delas são: tosse, congestão nasal, dores no peito e garganta, garganta irritada, dispneia, entre outros ${ }^{2}$. As doenças respiratórias constituem um problema de saúde pública, sendo uma das principais causas de internação no Sistema Único de Saúde (SUS). O uso do tabaco contribui para esse quadro: no Brasil, entre 1990 e 2015, as Doenças Pulmonares Obstrutivas Crônicas (DPOC) foram as principais causas de mortes relacionadas com o tabagismo ${ }^{3}$.

No período de 2003 a 2013, a taxa de mortalidade por Doenças Respiratórias Crônicas (DRC) no país foi de 32,6 para cada 100 mil habitantes. Entre as principais causas, estão: bronquite, enfisema, doenças crônicas obstrutivas e doenças do aparelho respiratório, totalizando $94,3 \%{ }^{4}$.

Esta investigação torna-se relevante, tendo em vista as taxas de afastamento ao trabalho devido a doenças do aparelho respiratório. Por exemplo, em estudo com 400 servidores públicos, 301 (75\%) sujeitos apresentaram pelo menos um dia de licença no trabalho; e, desses, 107 (35,79\%) sujeitos tiveram uma média de 8,4 dias de afastamento no trabalho por doenças do aparelho respiratório ${ }^{5}$.

A prova de função pulmonar é um recurso capaz de estimar alterações dos volumes e capacidades pulmonares e alterações da força muscular respiratória com o objetivo de caracterizar o padrão de normalidade da função pulmonar, diagnosticar, estimar a gravidade de doenças respiratórias e para elaboração de métodos preventivos de doenças respiratórias ${ }^{6-8}$.

Dada a importância da avaliação fisioterapêutica respiratória, por proporcionar parâmetros para estabelecer medidas preventivas efetivas para doenças respiratórias ocupacionais, $\mathrm{o}$ objetivo do estudo foi avaliar a capacidade respiratória de servidores de bibliotecas de uma universidade pública.

\section{METODOLOGIA}

\section{Amostra}

Estudo analítico observacional transversal, aprovado pelo Comitê de Ética em Pesquisa em Seres Humanos do Setor de Ciências da Saúde da Universidade Federal do Paraná (UFPR) pelo parecer $n^{\circ} 1175594$.

Considerando-se que a população de bibliotecários da UFPR é composta por 250 servidores, a amostra da primeira etapa do estudo foi constituída por 136 participantes, que responderam a dois instrumentos, cujos resultados serão publicados em outro artigo.

Dos 136 participantes da primeira etapa, 118 foram convidados a participar da segunda etapa do estudo. Foram excluídos 18 servidores (aqueles que eram oriundos de campus do interior do estado, devido à dificuldade de locomoção para a realização da avaliação). 
Contudo, não foi possível estabelecer contato e/ou não responderam ao convite via e-mail 80 servidores. Ademais, 6 deles não concordaram em participar da avaliação da capacidade respiratória. Assim, a avaliação foi realizada com 33 servidores, sendo excluídos 4 dos participantes por não concordarem em realizar todos os testes do protocolo de avaliação. Portanto, a amostra foi composta por 28 servidores de bibliotecas da UFPR (Figura 1).

O poder da amostra foi calculado no programa $\mathrm{G}^{\star}$ Power 3.1.3, considerando os seguintes critérios: tamanho de efeito 0,50 ; erro a 0,05 ; resultando no poder (1$\beta)$ de 0,72 .

Para a coleta de dados, cada servidor foi convidado a participar do estudo, comparecendo a uma avaliação no Laboratório de Fisioterapia no Setor de Ciências Biológicas, Campus Centro Politécnico da Universidade Federal do Paraná; no qual já haviam assinado o Termo de Consentimento Livre e Esclarecido (TCLE) de estudo preliminar.

\section{Instrumentos de avaliação}

Foi elaborada uma ficha de avaliação para o registro dos dados pessoais e dos resultados dos testes dos sujeitos da pesquisa.

A avaliação foi composta por manovacuometria, espirometria, teste de caminhada de 6 minutos e Escala de Borg Modificada.

A manovacuometria avalia a força muscular respiratória por meio da mensuração da pressão expiratória máxima (PEmáx), que avalia os músculos intercostais interno e transverso do tórax; e da pressão inspiratória máxima (PImáx), que avalia o músculo diafragma e os músculos inspiratórios ${ }^{6,9,10}$. No presente estudo, foi utilizado o equipamento manovacuômetro Wika.

A espirometria consiste em um teste que permite o diagnóstico e a quantificação dos distúrbios ventilatórios, por meio da análise de fluxo aéreos, volumes e capacidades pulmonares ${ }^{11}$. Para a

Figura 1. Etapas seguidas no estudo para seleção e inclusão/exclusão dos participantes

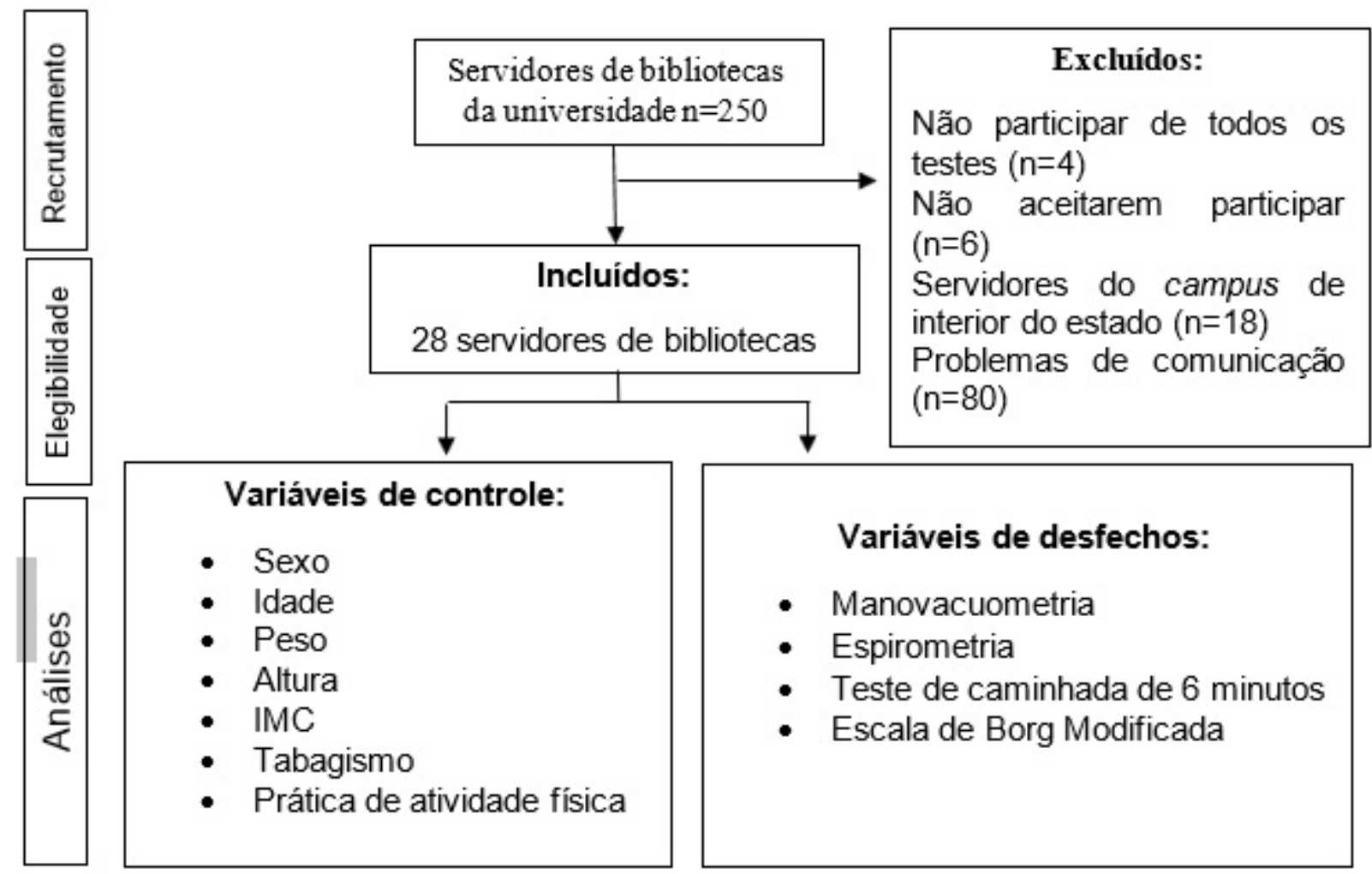


realização da espirometria, foi utilizado o espirômetro Spirobank G MIR com o programa Winspiropro.

O teste de caminhada de 6 minutos (TC6) é considerado submáximo visto que os indivíduos escolhem a sua própria intensidade de exercício. É utilizado para avaliar a resposta de um indivíduo ao exercício e propicia uma análise global do sistema cardiorrespiratório ${ }^{13}$.

Escala de Borg Modificada é uma escala de 10 pontos na qual a intensidade da dispneia sentida no momento da avaliação é graduada por meio de números que descrevem a intensidade da dispneia, desde "nenhuma falta de ar" até "falta de ar máxima". O sujeito deve informar qual número representa sua falta de $a^{14}$.

\section{Análise Estatística}

Foi realizada uma análise descritiva de dados, por meio de média, desvio padrão, máximo, mínimo, frequência absoluta e relativa. Para calcular os valores previstos individualmente de cada participante da manovacuometria, foi utilizada a fórmula segundo Neder ${ }^{15}$. No teste de caminhada de 6 minutos, foi utilizado a fórmula segundo Enright e Sherrill ${ }^{16}$ para calcular os valores previstos individualmente dos participantes. Para verificar a normalidade dos dados, foi utilizado o teste Shapiro-Wilk. Após esta verificação, foi utilizado o teste não paramétrico de Wilcoxon pareado para comparar se duas amostras dependentes são iguais. Para análise de tabelas de contingência, foi utilizado o Teste Exato de Fisher. Para avaliar a correlação, foi utilizada a correlação de Spearman que avalia a relação entre duas variáveis, sejam elas lineares ou não. Foi considerado significativo $\mathrm{p}$-valor $<0,05$. As análises foram feitas com o software estatistíco R e o SPSS 20.0 .

\section{RESULTADOS}

A média da idade dos servidores de biblioteca foi de $37,9( \pm 11,97)$ anos com predomínio de participantes do sexo feminino $(67,9 \%)$, a maioria com pósgraduação, sendo $85,7 \%$ não fumantes e $53,6 \%$ praticantes de atividade física. De acordo com a Classificação Organização Pan-Americana de Saúde (OPAS) ${ }^{17}$, a maioria dos participantes $(35,7 \%)$ foi classificada como obesos pelo índice de massa corporal (Tabela 1).

Apenas 9 participantes (32,14\%) tiveram a PEmáx adequada. E a PImáx de 5 participantes $(17,86 \%$, estavam adequadas de acordo com a fórmula de Neder ${ }^{15}$.

Foram analisados na espirometria VEF1, CVF, VEF1/CVF, FEF-25-75\%, PFE; e, ao correlacionar com a PImáx e com a PEmáx, apresentaram relação significativa para as variáveis PFE litros pré e pós-broncodilatador (correlação de Spearman $\mathrm{p}<0,05)$ (Tabela 1).

No quadro 2, na escala de dispneia de Borg inicial, 14,28\% dos participantes relataram intensidade de dispneia moderada a intensa pré-TC6; e após o TC6, a mesma intensidade foi relata por $35,71 \%$ dos participantes.

No teste de caminhada de 6 minutos, a distância média percorrida pelos participantes foi de 455,375 metros; e a média prevista, 557,165 metros. A maioria dos participantes $(82,14 \%)$ estava abaixo dos valores normais, pois não atingiram a distância prevista de acordo com a fórmula Enright e Sherrill ${ }^{16}$. Houve diferença entre a distância percorrida em metros e a distância prevista em metros (Teste Wilcoxon, $\mathrm{p}=0.00121$ ) (Figura 2). 
Tabela 1. Caracterização dos participantes da pesquisa.

\begin{tabular}{lcc}
\hline \multicolumn{1}{c}{ Variáveis } & $\mathbf{n}$ & $\%$ \\
\hline Sexo & & \\
\hline Masculino & 9 & $32,1 \%$ \\
Feminino & & $67,9 \%$ \\
\hline Grau de escolaridade & 12 & $42,9 \%$ \\
\hline Médio & 6 & $21,4 \%$ \\
Superior & 10 & $35,7 \%$ \\
Pós-graduação & & \\
\hline Índice de Massa Corpórea & 6 & $21,4 \%$ \\
\hline Baixo peso (IMC<23kg/m2) & 9 & $32,2 \%$ \\
Peso normal $(23<\mathrm{IMC}<28 \mathrm{~kg} / \mathrm{m} 2)$ & 3 & $10,7 \%$ \\
Pré-obesidade $(28<\mathrm{IMC}<30 \mathrm{~kg} / \mathrm{m} 2)$ & 10 & $35,7 \%$ \\
Obesidade $(\mathrm{IMC}>30 \mathrm{~kg} / \mathrm{m} 2)$ & & \\
\hline Prática de atividade física & 13 & $46,4 \%$ \\
\hline Não & 15 & $53,6 \%$ \\
Sim & & \\
\hline Tabagismo & 1 & $3,6 \%$ \\
\hline Sim & 24 & $85,7 \%$ \\
Não & 3 & $10,7 \%$ \\
\hline Ex-fumante & & \\
\hline
\end{tabular}

Tabela 2. Correlação entre a manovacuometria e a espirometria pré e pós administração de broncodilatador.

\begin{tabular}{lcccc}
\hline \multirow{2}{*}{ Variáveis } & \multicolumn{2}{c}{ PEMáx } & \multicolumn{2}{c}{ PIMáx } \\
\cline { 2 - 5 } Pré-broncodilatador & & p-valor & rho & p-valor \\
\hline VEF1 & 0,2441 & 0,262 & 0,2434 & 0,290 \\
CVF & 0,2191 & 0,210 & 0,2071 & 0,211 \\
VEF1/CVF & $-0,105$ & 0,595 & 0,0522 & 0,791 \\
FEF 25-75 & 0,2247 & 0,250 & 0,2728 & 0,160 \\
PFE & 0,4261 & $0,002^{*}$ & 0,4959 & $0,007^{*}$ \\
\hline Pós-broncodilatador & & & & \\
\hline VEF1 & 0,2572 & 0,153 & 0,1927 & 0,233 \\
CVF & 0,2769 & 0,186 & 0,2328 & 0,325 \\
VEF1/CVF & 0,0581 & 0,769 & 0,1781 & 0,364 \\
FEF 25-75 & 0,3197 & 0,097 & 0,373 & 0,060 \\
PFE & 0,5555 & $0,002^{*}$ & 0,4687 & $0,011^{*}$ \\
\hline
\end{tabular}

PEMáx: Pressão Expiratória Máxima; PIMáx: Pressão Inspiratória Máxima; VEF1: Volume Expiratório Forçado no $1^{\circ}$ segundo; CVF: Capacidade Vital Forçada; VEF1/CVF: Relação entre o Volume Expiratório Forçado no $1^{\circ}$ segundo e a Capacidade Vital Forçada; FEF 25-75: Fluxo Expiratório Forçado Médio; PFE: Pico de Fluxo Expiratório; rho: Coeficiente de Correlação de Spearman; ${ }^{\star}$ Estatisticamente significativo a nível de $5 \%$. 
Tabela 3. Avaliação do nível de esforço através da Escala de Dispneia de Borg Modificada nos momentos pré e pós intervenção

\begin{tabular}{cccc}
\hline Número & Intensidade & Borg inicial n (\%) & Borg final n (\%) \\
\hline 0 & Nenhuma & $11(39,29)$ & $4(14,29)$ \\
0,5 & Muito, muito leve & $4(14,29)$ & $1(3,57)$ \\
1 & Muito leve & $2(7,14)$ & $6(21,43)$ \\
2 & Leve & $7(25)$ & $7(25)$ \\
3 & Moderada & $2(7,14)$ & $3(10,71)$ \\
4 & Pouco intensa & - & $5(17,86)$ \\
5 & Intensa & $1(3,57)$ & $1(3,57)$ \\
6 & & $1(3,57)$ & $1(3,57)$ \\
7 & Muito intensa & - & - \\
8 & Muito, muito intensa & - & - \\
9 & Máxima & - & - \\
10 & & - & - \\
\hline
\end{tabular}

Figura 2. Resultados do teste de caminhada dos seis minutos

Teste de Caminhada de 6 minutos

25
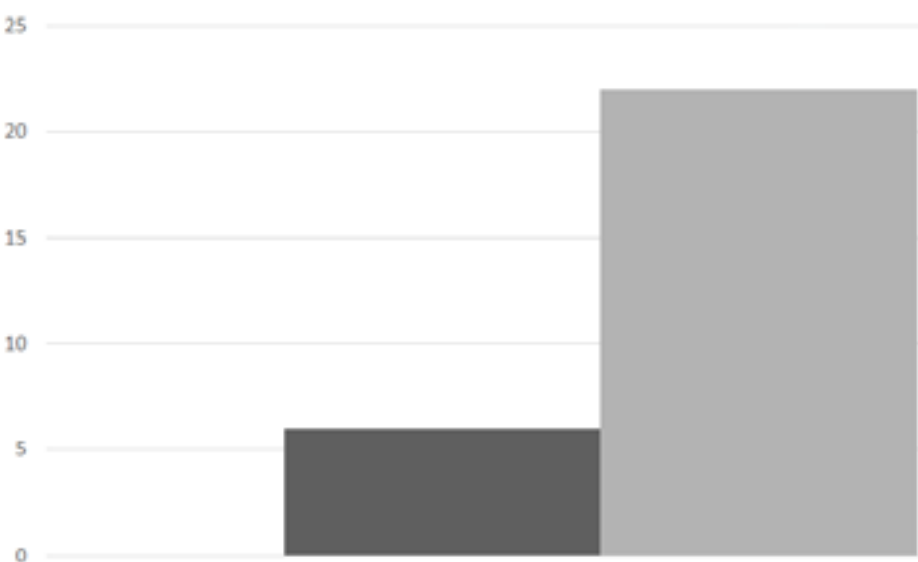

TC6

ADEQUADO INADEQUADO 


\section{DISCUSSÃO}

Os participantes do estudo são, em sua maioria, mulheres adultas (média de idade 37,92 anos), que, portanto, ainda não sofreram alterações da capacidade respiratória causada pela senescência, uma vez que o processo de envelhecimento gera alterações fisiológicas no sistema respiratório, influenciando na mecânica respiratória principalmente a partir dos $60 \operatorname{anos}^{18,19}$

A maioria dos participantes da pesquisa foi classificada como obesos, sendo assim, sugere-se que a obesidade está associada a uma diminuição da capacidade pulmonar, visto que a maioria não atingiu o previsto no teste da manovacuometria. Esses resultados estão de acordo com a literatura, na qual a força muscular respiratória de indivíduos com obesidade comparada com indivíduos eutróficos, em que os obesos apresentam menores valores da PImáx e PEmáx ${ }^{20-23}$.

Por outro lado, a prática de atividade física gera uma melhora na força muscular respiratória relacionada com PEmáx e PImáx, quando comparado a indivíduos sedentários ${ }^{24,25}$. Divergindo do presente estudo em que a maioria relatou realizar atividade física, no entanto, a maioria não atingiu o previsto na manovacuometria, por isso, questiona-se o tipo, intensidade, duração e frequência dos exercícios que estão sendo realizados.

Quando comparados valores da PImáx em indivíduos tabagistas e não tabagista, os não tabagistas têm valores maiores que a predita, e os tabagistas não alcançam a predita. Já na PEmáx, não há diferença significativa entre tabagistas e não tabagistas ${ }^{26,27}$. A maioria dos participantes do presente estudo não era fumante, portanto, sugere-se que o fato de não atingirem o previsto teve outra influência não sendo o tabagismo, por exemplo, a obesidade.

$\mathrm{Na}$ análise entre a espirometria e a manovacuometria, pode-se observar relação entre o PFE e a PImáx e PEmáx pré e pós-broncodilatador. Sendo que o máximo fluxo gerado durante a expiração forçada é dependente da força dos músculos expiratórios, o PFE está diretamente relacionado com a força muscular respiratória. Estudo realizado com 81 indivíduos saudáveis mostrou que houve influência da força dos músculos expiratórios no PFE de vias aéreas6.

A faixa etária entre 20 e 40 anos de idade é caracterizada por possuir melhor desempenho e capacidade funcional na avaliação do teste de caminhada de 6 minutos ${ }^{28,29}$. Entretanto, apesar da média de idade dos participantes situar-se em 37,92 anos, a maioria não atingiu a distância prevista, sugere-se então que outros fatores, não sendo a idade, prejudiquem o desempenho dos indivíduos no teste, inclusive questionase a veracidade das informações prestadas pelos participantes quanto à prática de atividade física, pois as expectativas eram de resultados melhores.

Estudo realizado com indivíduos saudáveis de 50 a 85 anos mostrou que o sexo feminino percorre uma distância menor no teste de caminhada de 6 minutos comparado ao sexo masculino ${ }^{30}$. A maioria dos participantes do presente estudo era do sexo feminino; sugere-se que esse fator pode influenciar o desempenho no teste de caminhada. 
Foi encontrado um menor desempenho no teste de caminhada de 6 minutos em indivíduos com IMC maior que $25 \mathrm{~kg} / \mathrm{m}^{2} 31,32$. No presente estudo, a maioria dos participantes foi classificada como obesos, apresentando um IMC $>30 \mathrm{~kg} / \mathrm{m}^{2}$; sugere-se que esse fator pode influenciar o desempenho no teste de caminhada. Já em outro estudo realizado com mulheres com média de IMC $43 \mathrm{~kg} /$ $\mathrm{m}^{2}$, não houve diferença significativa entre a distância percorrida no TC6 e a distância predita ${ }^{20}$.

\section{CONCLUSÃO}

Este estudo mostrou que o sexo feminino e a obesidade possuem relação para uma adequada capacidade respiratória. Outrossim, que a força muscular respiratória possui estreita relação com o pico de fluxo expiratório.

Sugere-se também que uma adequada prescrição de exercício físico pode influenciar na capacidade e função pulmonar do indivíduo.

É possível concluir que há necessidade da implementação de prevenção e promoção da saúde nos ambientes das bibliotecas relacionadas com o emagrecimento e com a saúde respiratória dos bibliotecários, para isso, é importante que surjam novos estudos a fim de contribuir para o planejamento de medidas preventivas.

\section{REFERÊNCIAS}

1. Malta DC, Moura L, Prado RR, Escalante JC, Schmidt MI, Duncan BB. Mortalidade por doenças crônicas não transmissíveis no Brasil e suas regiões, 2000 a 2011. Epidemiol Serv Saúde. Brasília 2014; 23(4):599-608.

2. Fonseca FR, Vasconcelos CH. Estudo da distribuição de doenças respiratórias no estado de Santa Catarina. Brasil Cad Saúde Colet. 2011; 19(4): 454-60.
3. José BPS, Corrêa RA, Malta DC, Passos VMA, França EB, Teixeira RA, et al. Mortalidade e incapacidade por doenças relacionadas à exposição ao tabaco no Brasil, 1990 a 2015. Rev Bras Epidemiol. 2017; 20: 75-89.

4. Boletim Epidemiológico. Secretaria de Vigilância em Saúde, Ministério da Saúde, Brasil. Vol. 47, 2016.

5. Andrade TB, Souza MGC, Simões MPC, Andrade FB. Prevalência de absenteísmo entre trabalhadores do serviço público. Scientia Medica. 2008; 18(4): 166-171.

6. Nagato AC, Nunes LAS, Dourado VA, Diniz MF, Silva, MAS, Dornelas $\mathrm{G}$, et al. Correlação entre a pressão expiratória máxima (PEmáx.) e pico de fluxo expiratório máximo (PFE) em indivíduos saudáveis. Rev interdisciplin estud exp anim hum. 2012; 4(único): 07-15.

7. Gulart AA, Santos K, Munari AB, Karloh M, Cani KC, Mayer AF. Relação entre a capacidade funcional e a percepção de limitação em atividades de vida diária de pacientes com DPOC. Fisioter Pesq. 2015; 2 (22): 104-111.

8. Borlee F, Yzermans CJ, Krop E, Aalders B, Rooijackers J, Zock J-P. Spirometry, questionnaire and electronic medical record based COPD in a population survey: Comparing prevalence, level of agreement and association swith potential risk factors. PLoS ONE. 2017; 3(12): 1-16.

9. Bessa EJC, Lopes AJ, Rufino R. A importância da medida da força muscular respiratória na prática da pneumologia. Pulmão. 2015; 24(1):374.

10. Simoes RP, Castello V, Auad MA, Dionisio J, Mazzonetto M. Prevalence of reduced respiratory muscle strength in institutionalized elderly people. Sao Paulo. Medical Journal. 2009; 127 (2): 78-83. 
11. Pereira CAC. Espirometria. J Pneumol. 2002; 28(Supl 3).

12. Morales-Blanhir JE, Vidal CDP, Romero MJR, Castro MMG, Villegas AL, Zamboni M. Teste de caminhada de seis minutos: uma ferramenta valiosa na avaliação do comprometimento pulmonar. J Bras Pneumol. 2011; 37(1):110-117.

13. Fernandes PM, Pereira NH, Santos ACBC, Soares EMSM. Teste de caminhada de seis minutos: avaliação da capacidade funcional de indivíduos sedentários. Rev Bras Cardiol. 2012; 25(3):185-191.

14. Brunetto AF, Paulin E, Yamguti WPS. Comparação entre a escala de Borg modificada e a escala de Borg modificada análogo visual aplicadas em pacientes com dispneia. Rev Bras Fisioter. 2002; 6: 41-45.

15. Neder JA, Andreoni S, Lerario MC, Nery LE. Reference values for lung function tests. II. Maximal respiratory pressures and voluntary ventilation. Braz J Med Biol Res. 1999; 32(6): 71927.

16. Enright PL, Sherril DL. Reference equations for the six-minute walk in healthy adults. Am J Respir Crit Care Med.1998; 158(5 Pt 1):1384-7.

17. SABE - Saúde, Bem-estar e Envelhecimento. Lebrão, M. L.; Duarte, Y. A. O. O Projeto Sabe no município de São Paulo: uma abordagem inicial. Brasília: Organização Pan - Americana da Saúde; 2003.

18. Ruivo S, Viana P, Martins C, Baeta C. Efeito do envelhecimento cronológico na função pulmonar. Comparação da função respiratória entre adultos e idosos saudáveis. Rev Port Pneumol. 2009; XV(4): 629-653.
19. Pascotini FS, Fedosse E, Ramos MC, Ribeiro VV, Trevisan ME. Força muscular respiratória, função pulmonar e expansibilidade toracoabdominal em idosos e sua relação com o estado nutricional. Fisioter Pesqui. 2016; 23(4):416-422.

20. Domingos-Benício NC, Gastaldi AC, Perecin JC, Avena KM, Guimarães RC, Sologuren MJJ, et al. Medidas espirométricas em pessoas eutróficas e obesas, nas posições ortostatícas, sentada e deitada. Ver Assoc Med Bras. 2004; 50(2):142-7.

21. Ribeiro GF, Araújo LMB, SouzaMachado A, Ribeiro PA. Avaliação da função pulmonar em indivíduos obesos assintomáticos respiratórios: correlação entre dados antropométricos e espirométricos. Rev Bras Alerg Imunopatol. 2007; 30(6):227-31.

22. Soares KKD, Gomes ELFD, Júnior AB, Oliveira LVF, Sampaio LMM, Costa D.; Avaliação do desempenho físico e funcional respiratório em obesos. Fisioter Mov. 2011; 24(4):697-704.

23. Melo LC. Silva MAM, Calles ACN. Obesidade e função pulmonar: uma revisão sistemâtica. Einstein. 2014; 12:120-5.

24. Leal AH, Hamasaki TA, Jamami M, DI Lorenzo VAP, Pessoa BV. Comparação entre valores de força muscular respiratória medidos e previstos por diferentes equações. Fisioter pesqui.2007;14(3):25-30.

25. Gonçalves MP, Tomaz CAB, Cassiminho ALF, Dutra MF. Avaliação da força muscular inspiratória e expiratória em idosas praticantes de atividade física e sedentárias. $\mathrm{R}$ bras $\mathrm{Ci}$ e Mov. 2006; 14(1): 37-44. 
26. Macedo LB, Ormond LS, Santos LLAG, Macedo MC. Tabagismo e força muscular respiratória em adultos. ASSOBRAFIR Ciência 2011; 2(2):9-18.

27. Freitas ERFS, Araujo ECLS, Alves KS. Influência do tabagismo na força muscular respiratória de idosos. Fisioter Pesq. 2012; 19(4):326-331.

28. Botelho C, Barbosa G, Jardim JRB. Sintomas respiratórios, espirometria e tabagismo em adultos.

J pneumol.1989; 15(2): 74-78.

29. Neves DD, Campos H, Pereira FS, Santos ND, Brito VAD, Souza TFA, Dias RM. Tabagismo e função pulmonar em programas de busca de doentes com doença pulmonar obstrutiva crônica (DPOC). Pulmão. 2005;14(4):294-299.
30. Sperandio EF, Arantes RL, Matheus AC, Silva RP, Lauria, VT, Romiti $\mathrm{M}$, et al. Distúrbio ventilatório restritivo sugerido por espirometria: associação com risco cardiovascular e nível de atividade física em adultos assintomáticos. J Bras Pneumol. 2016; 42(1): 22-28.

31. Troosters T, Gosselink R, Decramer M. Six minute walking distance in healthy elderly subjects. Eur Respir J. 1999;14: 270-4.

32. Pires SR, Oliveira AC, Parreira VF, Britto RR. Teste de caminhada de seis minutos em diferentes faixas etárias e índices de massa corporal. Rev bras Fisioter. 2007; 11(2): 147-151. 